\title{
Nonlinear Simulation of Edge-Localized Mode in Spherical Tokamak
}

\author{
N. Mizuguchi et al.
}




\title{
Nonlinear Simulation of Edge-Localized Mode in Spherical Tokamak
}

\author{
N. Mizuguchi 1)2), R. Khan 2), ${ }^{1}$ T. Hayashi 1)2), N. Nakajima 1)2) \\ 1)National Institute for Fusion Science, Toki, Japan \\ 2)Department of Fusion Science, Grad. Univ. Advanced Studies, Toki, Japan \\ e-mail contact of main author: mizu@nifs.ac.jp
}

\begin{abstract}
A numerical modeling for the dynamics of an edge-localized mode(ELM) crash in the spherical tokamak is proposed with a consecutive scenario which is initiated by the spontaneous growth of the ballooning mode instability by means of a three-dimensional nonlinear magnetohydrodynamic simulation. The simulation result shows a two-step relaxation process which is induced by the intermediate-n ballooning instability followed by the $m / n=1 / 1$ internal kink mode, where $m$ and $n$ represent the poloidal and toroidal mode numbers, respectively. By comparing with the experimental observations, we have found that the simulation result can reproduce several characteristic features of the so-called type-I ELM in an appropriate time scale:(1)relation to the ballooning instability, (2)intermediate- $n$ precursors, (3)low- $n$ structure on the crash, (4)formation and separation of the filament, and (5)considerable amount of loss of plasma. Furthermore, the model is verified by examining the effect of diamagnetic stabilization and comparing the nonlinear behavior with that of the peeling modes. The ion diamagnetic drift terms are found to stabilize some specific components linearly; nevertheless they are not so effective in the nonlinear dynamics such as the filament formation and the amount of loss. For the peeling mode case, no prominent filament structure is formed in contrast to the ballooning case.
\end{abstract}

\section{Introduction}

The edge-localized mode(ELM) is a repetitious instability which is often observed in the pedestal region of the H-mode operations of toroidal devices. Although ELMs are slightly harmful to the edge confinement, they also play an essential role for impurity control to sustain the H-mode state. Therefore, to control the ELMs is one of the most important issues for the advanced operations of toroidal plasmas. In recent spherical tokamak(ST) experiments, as well as conventional large tokamaks, several "types" of the ELMs are also observed as the confinement properties and the measurement environment developed. Not only the most commonly observed type-I ELM, which has larger fluctuation level than other types of ELM, but also several kinds of small ELMs are observed. Some of them have unique characteristics to the ST devices. Moreover, the geometrical compactness of the ST configuration has been helpful in understanding the global structure of ELMs. Especially, detailed filamentary structures on the plasma surface are clearly observed in the MAST[1] and NSTX[2].

Numerous linear stability analyses have shown that the onset of the observed type-I ELMs can be well explained by the peeling-ballooning mode instabilities[3]. Namely, the large pressure gradient in the pedestal region can drive the ideal ballooning modes, and the large edge bootstrap current due to the steep density gradient on the edge can destabilize the external kink modes, i. e., the peeling modes. The experimental evidences that there is a close relation between the operation regime and the stability limits of those modes support the model that ELMs are excited by the combination of both modes.

${ }^{1}$ Prof. T. Hayashi regrettably passed away during this work on April 13, 2006. 
On the other hand, the physical models for the nonlinear behavior of the ELMs are insufficient at present. Experimentally, several prominent filamentary structures extending helically along the magnetic field, the convective loss of plasma, and the post-cursory deformations with low- $n$ components are observed in the ELM crash phase. These nonlinear behaviors are linked directly to the performance of the H-mode state.

In this paper, we propose a modeling for the nonlinear dynamics of an ELM crash with a consecutive scenario which is initiated by the spontaneous growth of the ballooning mode instability by means of a magnetohydrodynamic (MHD) simulation. In Sec. 2, the numerical model is explained. The overall simulation result is described in Sec. 3. Several considerations to the result with the effect of diamagnetic stabilization and the comparison with that of the peeling mode are discussed in Sec. 4. The model is summarized in Sec. 5.

\section{Simulation Model}

The numerical model of the simulation is based on the nonlinear resistive compressive MHD equations,

$$
\begin{aligned}
& \frac{\partial \rho}{\partial t}=-\nabla \cdot(\rho \mathbf{v}) \\
& \frac{\partial(\rho \mathbf{v})}{\partial t}=-\nabla \cdot(\rho \mathbf{v v})-\nabla p+\mathbf{j} \times \mathbf{B}+\mu\left(\nabla^{2} \mathbf{v}+\frac{1}{3} \nabla(\nabla \cdot \mathbf{v})\right) \\
& \frac{\partial \mathbf{B}}{\partial t}=-\nabla \times \mathbf{E} \\
& \frac{\partial p}{\partial t}=-\nabla \cdot(p \mathbf{v})-(\gamma-1)\left(p \nabla \cdot \mathbf{v}+\eta j^{2}+\Phi\right)
\end{aligned}
$$

where $\rho, \mathbf{v}, \mathbf{B}$, and $p$ are the density, the flow velocity, the magnetic field, and the pressure, respectively. These variables are treated in normalized form, where the Alfven velocity at the magnetic axis becomes 1 . The current $\mathbf{j}$ and the electric field $\mathbf{E}$ are determined by

$$
\begin{aligned}
& \mathbf{j}=\nabla \times \mathbf{B}, \\
& \mathbf{E}=-\mathbf{v} \times \mathbf{B}+\eta \mathbf{j} .
\end{aligned}
$$

The resistivity $\eta$ and the viscosity $\mu$ is given as a uniform constant. $\Phi$ is the the viscous heating by which the total energy is locally conserved everywhere. The simulation is executed by using a finite-difference / Runge-Kutta method in a full toroidal three-dimensional geometry. The system includes the external open field, and a single-null $\mathrm{X}$ point. A perfect conducting wall located close to the plasma surface limits the boundary condition of the simulation. The initial condition is given by a reconstructed equilibrium from the NSTX, where $\beta_{0}=28 \%$, $q_{0}=0.89$, and $A=1.4$. The system is linearly stable for the ideal modes, but weakly unstable for the resistive ballooning mode. This numerical model has been already reported in our previous paper[4]. Although no ELM activity is observed in this shot, we adopt these conditions as a typical example of a marginally stable ST plasma for the resistive ballooning mode. The density is set to be uniformly $\rho=1$, and the vacuum region is treated as a low pressure plasma continuously from the core region, for simplicity. 
(a)

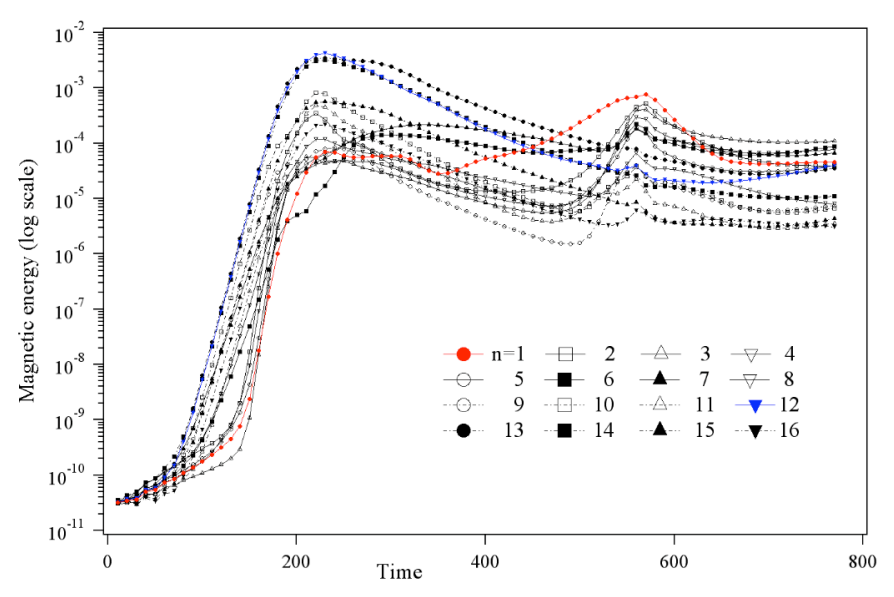

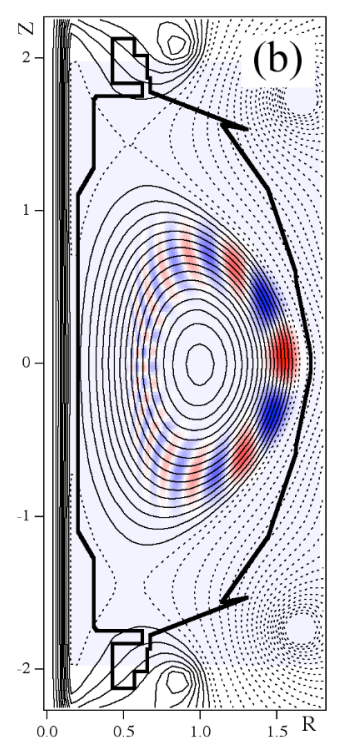

FIG. 1. (a)Time development of energy for each toroidal mode. (b)Poloidal mode structures for the $n=12$ mode.

\section{Overall Scenario of an ELM crash}

As reported in Ref.[4], the simulation result shows a two-step relaxation process induced by the intermediate- $n$ resistive ballooning mode followed by the $m / n=1 / 1$ internal kink mode, where $m$ and $n$ are the poloidal and toroidal mode numbers, respectively. Figure 1(a) shows the time development of the magnetic energy for each toroidal mode for the case where the normalized resistivity $\eta=1 \times 10^{-5}$. The simultaneous linear growth of several intermediate- $n$ modes can be seen until $t=200 \tau_{\mathrm{A}}$. Shown in Fig. $1(\mathrm{~b})$ is the poloidal mode structures of the $n=12$ mode, which clearly shows the typical ballooning nature, where the mode structure is highly localized in the low-field side. After the saturation of these modes at $t=360 \tau_{\mathrm{A}}$, only the $n=1$ mode begins to grow again, and ends in the internal kink relaxation. Thus, the simulation result has reproduced consecutively both the intermediate- $n(\sim 8-12)$ modes and the single $n=1$ deformation.

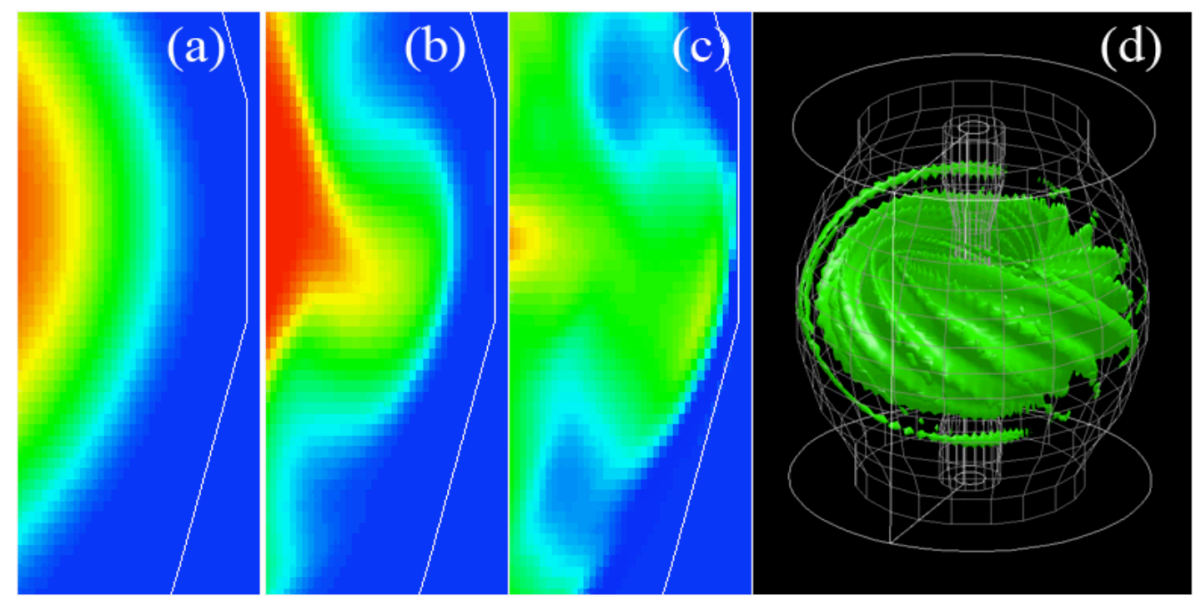

FIG. 2. (a)Pressure near the surface at $t=10 \tau_{t},(b) 230 \tau_{t},(c) 270 \tau_{s}$, and (d) $270 \tau_{A}$ in $3 D$. 
At around $t=200 \tau_{\mathrm{A}}$, several numbers of thin and elongated ridges are formed almost along the magnetic field lines on the plasma surface. They eventually turn into bubbles isolated from the core plasma. Such behavior is shown in Fig.2. Figures 2(a)-(c) show the contour of the pressure in a clipped poloidal plane near the plasma surface, on which a plasmoid is separated from the core. This behavior well agrees with the experimental observation in MAST[1]. The three-dimensional structure is shown in Fig. 2(d). One can see a floating thin filamentary structure and several ridges along the field lines, which are also in good agreement with the camera image taken in MAST. Especially, it should be noted that only a few numbers of filaments emerges prominently despite the toroidal mode number of the most dominant linear eigenmode is much larger. This fact can be explained by the toroidal mode couplings among multiple linear modes which exist close to each other.

The lifted plasmoid can carry part of the plasma out of the torus within itself . Moreover, under such a situation, a kind of magnetic reconnections can be induced by the same mechanism as the internal reconnection event in ST[5]. Namely, the plasma on the ridges is pushed against the external open magnetic field as the plasmoid grows. Then, strong current sheet structures can be formed along the ridges. These current sheet structure with a strong perpendicular plasma flow would drive a forced reconnection between the internal and external magnetic fields. The occurrence of the magnetic reconnection in the simulation result can be confirmed by tracing the magnetic field lines, which start from an external position. Some of the field lines go across the separatrix into the torus. Once such a reconnection occurs, a steep pressure gradient is formed abruptly along the reconnected field lines. This parallel pressure gradient can be another cause of the fast loss of the plasma. Such parallel plasma flows are also observed in the latter stage of the simulation result.

The poloidal pressure profile changes into a peaked one from a broader one in the central region due to the convection motions of the ballooning modes. The safety factor profile also goes below 1 at the center. These changes can induce another instability after $t=360 \tau_{\mathrm{A}}$, i. e., the $m / n=1 / 1$ internal kink mode. The system crashes once again due to the $1 / 1$ mode, and then recovers to a broader profile, just like the well-known sawtooth crash mechanism. During this process, one can see a characteristic non-axisymmetric structure with a large $n=1$ component, which is also clearly observed in NSTX[2] as a post-cursory deformation on a large ELM.
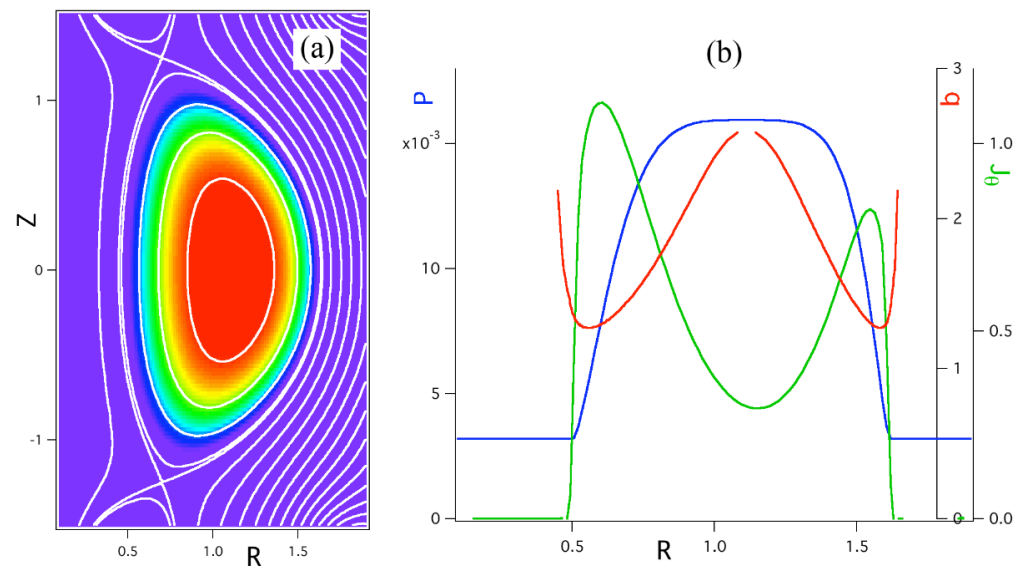

FIG. 3. The initial condition for the test case. (a) Poloidal flux(contours) and pressure(color). (b)Radial pressure(blue), toroidal current(green), and safety factor(red) profiles. 
Thus, our simulation result reproduces several characteristic features of a so-called type-I ELM in STs:(1)relation to the ballooning instability (2)intermediate- $n$ precursors (3)low- $n$ post-cursor (4)formation and separation of the filamentary structures (5)considerable amount of convective loss. As for the time scale, the simulation result is consistent with the experimental ELM rise times of the order of $\sim 100 \mu \mathrm{sec}$.

\section{Discussion}

\subsection{Comparison between the Peeling and Ballooning Modes}

As described in Sec.1, the ELMs are thought to be induced by not only the ballooning mode, but also the combination of the peeling mode. Especially, the smaller types of ELMs may have much relation to the peeling instability[6]. We have demonstrated in Sec.3 that the characteristic features of the type-I ELM can successfully reproduced with the nonlinear time development of a ballooning mode. In this section, we examine the nonlinear behavior driven by the peeling mode instabilities. To deal with the peeling modes, we compose another set of the initial and the boundary conditions with a numerical equilibrium which is obtained by solving the Grad-Shafranov equation under certain assumption for the pressure and the current profiles. To model the increased edge current, we assume polynomial functions for both the pressure and the current, in which the current is increased in the region where the pressure gradient becomes steep. Moreover, the configurations have the double-null separatrix and the far-off conducting boundaries to treat the external modes consistently, and the triangularity to suppress the unfavorable ballooning modes. By using a free-boundary Grad-Shafranov code, we have obtained such an ST equilibrium, as shown in Fig. 3. The poloidal flux is shown in Fig. 3(a), together with the pressure contours. The radial profiles are plotted in Fig. 3(b), where the pressure is broad, the current is highly hollow, and the safety factor is deeply negativesheared. The parameters for this equilibrium are $\beta_{0}=25 \%, q_{\min }=1.27$, and $A=2.0$.
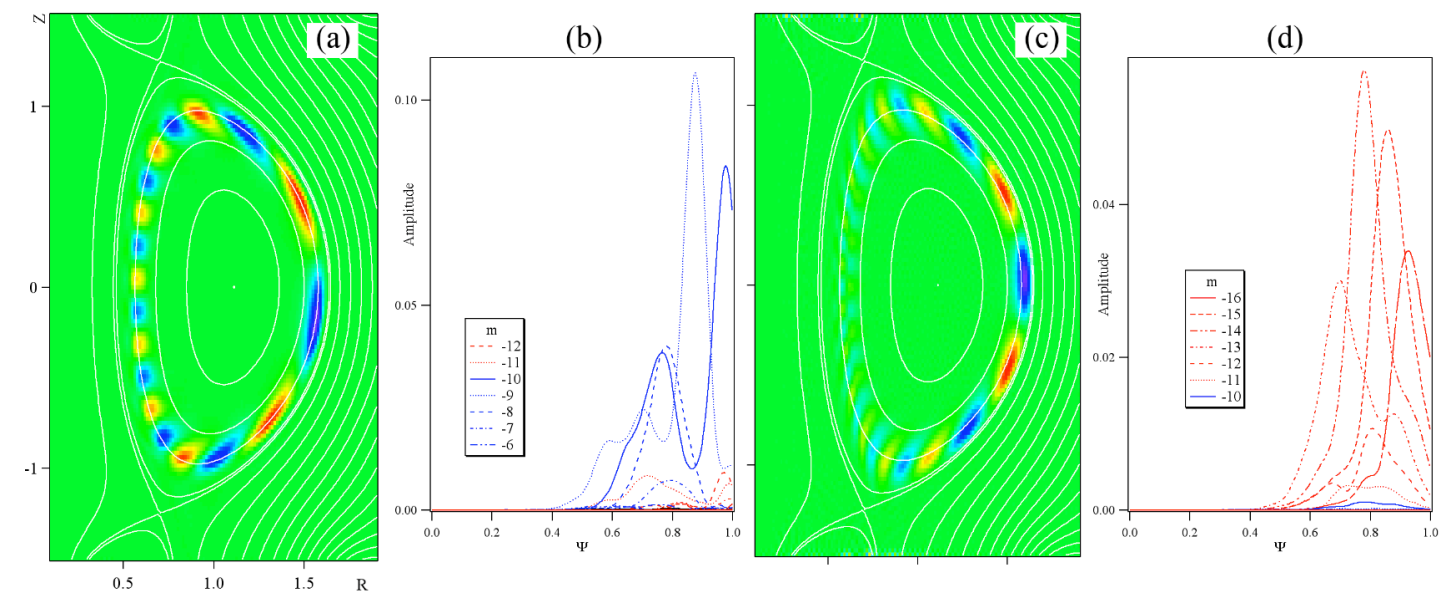

FIG. 4. (a)Linear eigenfunction for the $n=7$ peeling mode with the perturbations in pressure. (b)Poloidal expansion of magnetic energy for (a). (c)(d) Same as (a)(b) but for the $n=10$ ballooning mode. 

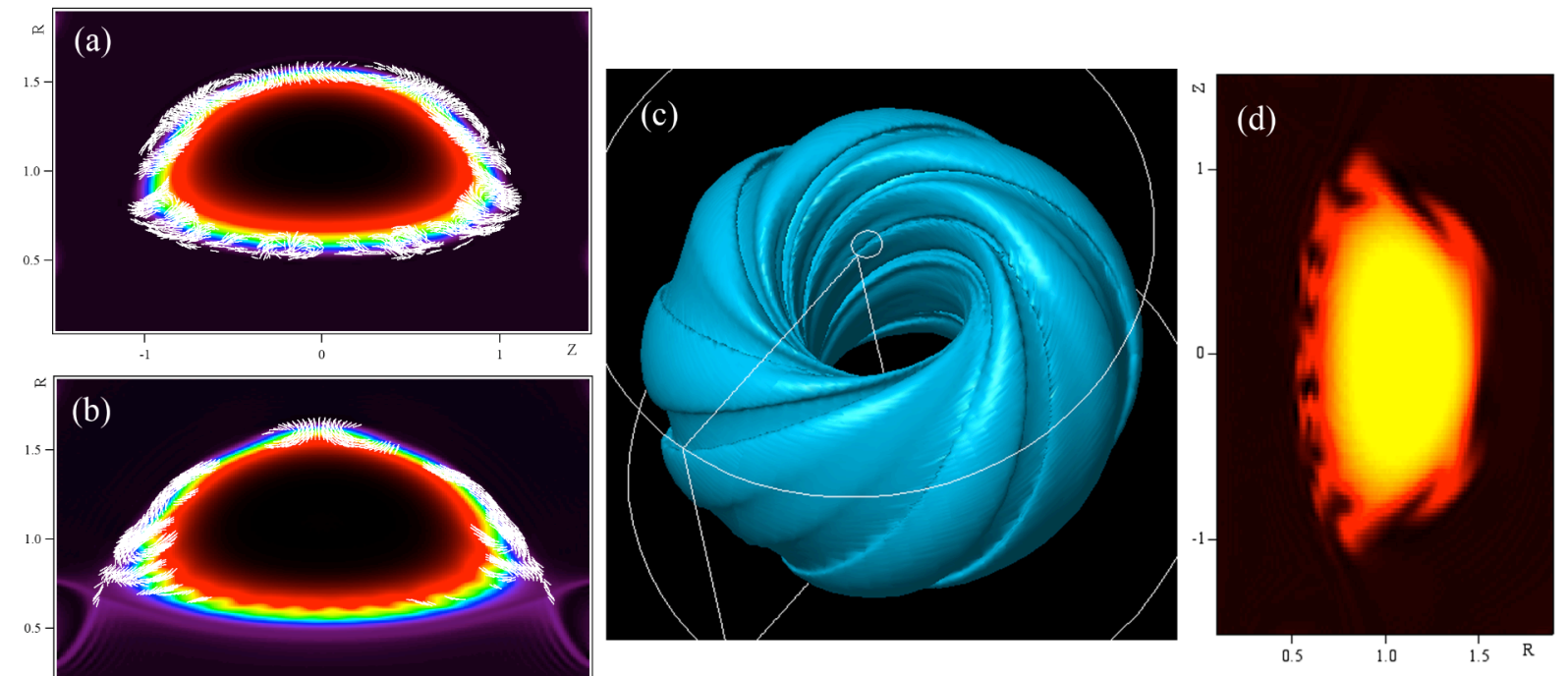

FIG. 5. (a) poloidal flow pattern of the peeling mode. (b) Same as (a) but for the ballooning mode. (c)3D structure of pressure for the peeling mode. (d) Pressure in the poloidal cross section.

The initial equilibrium is linearly unstable for some toroidal Fourier components within the ideal regime, in which the resistivity is small enough $\left(\eta<10^{-7}\right)$. The most dominant one is the $m / n=9 / 7$ component, which is excited near the minimum- $q$ surface. The poloidal mode structures are shown in Fig. 4(a) and (b). One can see that the mode shows typical characteristics of the peeling mode in that the mode structures are not poloidally localized and are extending toward the edge without significant poloidal mode couplings. Further analysis for the potential energy by using the energy principle shows a dominance of the current driven term, which supports the insight. On the other hand, with a larger resistivity $\eta=4 \times 10^{-5}$, the system becomes more unstable for the resistive ballooning mode, as well as the case described in Sec. 3 . The mode structures of the most dominant $n=10$ component are shown in Fig. 4(c) and (d). The structure shows typical ballooning nature as described in Sec. 3, in contrast to Fig. 4(a) and (b). Thus, we have obtained a pair of test cases of the peeling and the ballooning modes.

The nonlinear behavior is firstly directed by the plasma flow structures of the linear eigenfunctions. For the ballooning mode, the filamentary structure is formed by a radial convection motion in the edge of the low-field side, as described in Sec. 3. One can clearly see such poloidal flow patterns in the early nonlinear stage of the test case of the ballooning mode, shown in Fig.5(b). For the peeling case, on the other hand, the vortices become poloidally, and also toroidally, elongated near the surface, as shown in Fig. 5(a). Namely, the radial component is not so large that no prominent filamentary structure can form in the nonlinear stage. In stead of the filaments, the outermost layer of the plasma is "peeled" with a certain width, as shown in Fig. 5(c) and (d). This behavior of the peeling mode may induce a milder loss of plasma, which would be related to the smaller types of the ELM.

\subsection{Diamagnetic Stabilization of some Linear Modes}

We have also executed the drift model simulation to follow the dynamics under more realistic situations. According to the Hazeltine and Meiss model[7], we introduce the flow velocity 
variable as the sum of the conventional MHD velocity and the ion diamagnetic drift veloc$\operatorname{ity}\left(\mathbf{v}_{p}=\frac{m}{2 e} \frac{\mathbf{B} \times \nabla p}{\rho B^{2}}\right)$. The electron dynamics and the density gradient are neglected for simplicity. The result shows that the mode structures rotate both toroidally and poloidally with $v_{p} \sim 0.01 v_{A}$. Under the realistic parameters, the effect of the diamagnetic modification is small enough not to change the linear growth rate, as shown in Fig. $6(\alpha=1)$. However, if we use twice large value for $v_{p}(\alpha=2)$, which corresponds to the case with a smaller device dimension, the lower- $n$ modes are linearly stabilized, whereas the higher- $n$ modes remain unstable. It can be thought that the velocity shear formed by the diamagnetic effect suppresses the growth of the low- $n$ modes. The nonlinear dynamics, on the other hand, is not so affected by those modifications, since the nonlinear dynamics is dominated by the higher- $n$ components in this case.

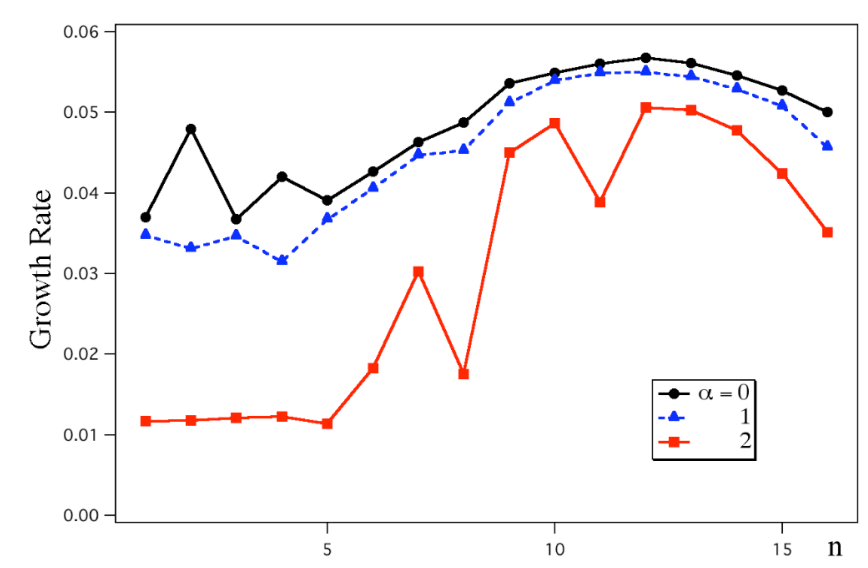

FIG. 6. Growth rate for each toroidal mode. $\alpha$ indicates the factor for the diamagnetic effect.

The diamagnetic stabilization effect may be sensitive to the profile, especially, the pressure gradient in the unstable region. More systematic scans for the diamagnetic effect in the broader core and steeper edge profile have been carried out with a simpler model, where only the diamagnetic rotation term is included in the advection term of the momentum equation as the lowest order modification[8]. The result again shows the less effect of the diamagnetic term with a realistic parameter. In this case, the stabilizing effect is observed in the higher- $n$ components with some increased value for $v_{p}$, mainly because the velocity shear exists only in the edge region. Therefore, the dominant toroidal mode number of the linear growth is shifted lower as the stabilization effect becomes larger. However, in the nonlinear stage, it should be noted that only a few filaments are universally formed in any case due to the toroidal mode couplings. Thus, our model is found to be sound against the diamagnetic stabilization effect.

\section{Concluding Remarks}

In this paper we have demonstrated the dynamics of ST plasma on a pulse of the ELM crash by means of a three-dimensional nonlinear simulation. A modeling for the filament formation on an ELM crash based on the nonlinear development of a ballooning mode was proposed. A consecutive scenario was obtained including the stages from the precursory intermediate- $n$ ballooning mode to the post-cursory $n=1$ deformation through the formation of a few filamentary structures. The impact on the result with the different driving source of the linear mode 
and the diamagnetic stabilization was examined. It has turned out that the ballooning nature is essential for the formation mechanism of the observed filamentary structure. Our numerical modeling with the global geometry which include the separatrix and the core region have revealed several unknown features such as the loss mechanism through the magnetic reconnection between the internal and external field, and the multi-step relaxation ranging over whole region of the torus. Quantitative analyses with more realistic configurations and the inclusion of the transport physics into the model are our future works.

\section{Acknowledgment}

The authors would like to thank Dr. S. Kaye, Dr. F. Paoletti, and Dr. S. Sabbagh for providing the reconstruction data, Dr. T. Ozeki and Dr. N. Aiba for their fruitful comments. This work is performed with the support and under the auspices of the NIFS Collaborative Research Program NIFS06KTAT034 and NIFS06KNXN072, and in part by the Grant-in-Aid from the Japanese Ministry of Education, Culture, Sports, Science, and Technology, Nos. 15760628 and 15540386. The numerical calculations are performed on the Plasma Simulator and the LHD Numerical Analysis System of NIFS.

\section{References}

[1]A.Kirk et al., Phys. Rev. Lett. 92 (2004) 245002.

[2]R. Maingi et al., Nucl. Fusion 45 (2005) 1066.

[3]P. Snyder et al., Phys. Plasmas 9 (2002) 2037.

[4]T. Hayashi, N. Mizuguchi et al., 19th IAEA-FEC, Lyon (2002) IAEA-CN-94/ TH/6-3.

[5]N. Mizuguchu, T. Hayashi, and T. Sato, Phys. Plasmas 7 (2000) 940.

[6]ASDEX team, Nucl. Fusion 29 (1989) 1959.

[7]R.D. Hazeltine and J.D. Meiss, Plasma Confinement (Dover, New York, 2003).

[8]R. Khan, N. Mizuguchi, et al., submitted to Phys. Plasmas. 